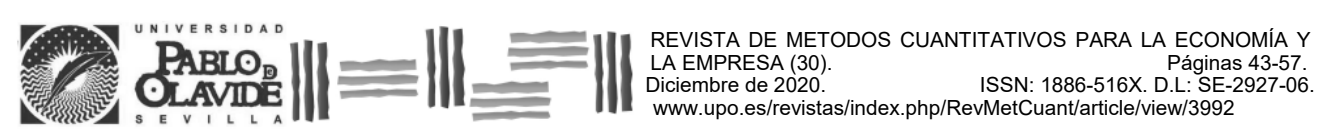

\title{
Industria 4.0 y la digitalización hacia la satisfacción laboral de las organizaciones en Tampico, Tamaulipas, México
}

\author{
DíAZ MARTÍNEZ, MARCO ANTONIO \\ Área de Posgrado e Investigación. Instituto Tecnológico Superior de Pánuco (Veracruz) \\ Correo electrónico: marco.diaz@itspanuco.edu.mx \\ ROMÁN SALINAS, REINA VERÓNICA \\ Área de Ingeniería Industrial. Instituto Tecnológico Superior de Pánuco (Veracruz)* \\ Correo electrónico: reina.roman@itspanuco.edu.mx \\ Santiago Santiago, Ariadna DaUlet \\ Área de Gestión Empresarial. Instituto Tecnológico Superior de Pánuco (Veracruz) \\ Correo electrónico: Ariadna.santiago@itspanuco.edu.mx \\ MEJía BARRIOS, CATALINA* \\ Correo electrónico: catalina.mejia@itspanuco.edu.mx \\ ZÁRATE CRUZ, RICARDO* \\ Correo electrónico: ricardo.zarate@itspanuco.edu.mx
}

\section{RESUMEN}

La industria 4.0 está relacionada en cada lugar y con el uso y la implementación de nuevas tecnologías para la mejora continua de los procesos administrativos. Como parte del crecimiento de una organización, es importante que día a día se adapte a los cambios tecnológicos que afectan las operaciones de los trabajadores o la seguridad laboral. Algunos de los elementos que abarca esta industria son el uso de equipos autónomos, robótica, simuladores de procesos, impresoras 3D, inteligencia artificial y equipos que comparten información en tiempo real. El objetivo de este artículo consiste en valorar los procesos de digitalización de las organizaciones de la ciudad de Tampico, Tamaulipas (México), desde el punto de vista del usuario, para identificar los factores determinantes de la satisfacción laboral. Se emplea la técnica multivariante de regresión de mínimos cuadrados parciales, considerando como factores de análisis de digitalización y su relación con el modelo de relación y colaboración, habilidades y competencias profesionales, formación digital y procesos de digitalización. Los resultados muestran que el grado de motivación aumenta con el incremento en la digitalización de los procesos y que la formación digital y las competencias profesionales necesitan aumentar gradualmente para tener un impacto positivo en relación con los procesos de digitalización.

Palabras clave: Industria 4.0, mejora continua, condiciones organizacionales, alfa de Cronbach.

Clasificación JEL: C4; C5; L00; L7; L80; L84.

MSC2010: 90B70; 62P30. 


\title{
Industry 4.0 and digitization towards job satisfaction of organizations in Tampico, Tamaulipas, Mexico
}

\begin{abstract}
Industry 4.0 is related in each place and with the use and implementation of new technologies for the continuous improvement of administrative processes. As part of the growth of an organization, it is important that day by day it adapts to technological changes that affect worker operations or job security. Some of the elements that this industry encompasses are the use of autonomous equipment, robotics, process simulators, 3D printers, artificial intelligence and equipment that share information in real time. The objective of this article is to assess the digitization processes of organizations in the city of Tampico, Tamaulipas (Mexico), from the user's point of view, to identify the determining factors of job satisfaction. The multivariate technique of partial least squares regression (or PLS, by Partial Least Squares (or SEM, by Structural Equation Models) is used, considering as digitization analysis factors and their relationship with the relationship and collaboration model, skills and competencies professionals, digital training and digitization processes. The results show that the degree of motivation increases with the increase in the digitization of processes and that digital training and professional competencies need to increase gradually to have a positive impact in relation to the processes of digitization.
\end{abstract}

Keywords: Industry 4.0, continuous improvement, organizational conditions, Cronbach's alpha. JEL classification: C4; C5; L00; L7; L80; L84.

MSC2010: 90B70; 62P30.

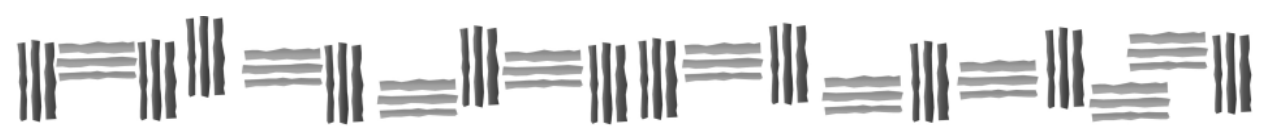




\section{Introducción.}

En el campo de los procesos de fabricación, avances en ciencia y tecnología apoyan continuamente el desarrollo de la industrialización en todo el mundo (Belvedere, Grando \& Bielli, 2013). En una revolución industrial desde una perspectiva de evolución tecnológica, se han identificado cuatro etapas comunes (Maynard, 2015; Kagermann, 2013), en las que los primeros tres tomaron alrededor de dos siglos y son el resultado, respectivamente de: 1) la introducción de agua y vapor en el funcionamiento de los procesos mecánicos; 2) la aplicación de tecnologías de producción en masa y 3) la combinación de electrónica y tecnologías de la información (TI) para generar un respaldo hacia la automatización de la fabricación (Drath \& Horch, 2014). En la actualidad, la atención al Internet de las cosas (IoT) y sus intervenciones en procesos industriales, así como a los ciber-sistemas (CPS), gobiernos e industrias de todo el mundo han notado esto (Atzori et al., 2010; Khaitan \& Mccalley, 2015). Esta tendencia ha actuado para beneficiarse de lo que esta nueva revolución industrial podría proporcionar (Ridgway et al., 2013).

\subsection{Planes y perspectivas de gobierno.}

Desde 2011, el gobierno de los Estados Unidos dio un comienzo muy importante hacia los debates, las acciones y las recomendaciones a nivel nacional y lo llamó "Asociación de Manufactura Avanzada (AMP)" para que Estados Unidos esté preparado para liderar la próxima generación de fabricación (Reif et al., 2014). Por otro lado, en 2012, un plan de acción llamado "Estrategia de alta tecnología 2020" se aprobó en Alemania y establece montos millonarios para el desarrollo de nuevas tecnologías de vanguardia. Como uno de los proyectos de este plan en el futuro es el de "Industria 4.0", que representa para el pueblo alemán una de las grandes ambiciones en el sector manufacturero (Kagermann et al., 2013).

En 2013, el gobierno francés comenzó una estrategia llamada "La Nouvelle France Industrielle" con 34 iniciativas sectoriales como las prioridades de la política industrial de Francia (Consell national de I'ndustrie, 2013). El gobierno de Singapur ha comprometido \$19 mil billones para su plan de investigación e innovación RIE 2020 (National Research Foundation, 2016). En 2013, el gobierno del Reino Unido presentó una imagen a largo plazo para su sector manufacturero hasta el año 2050 denominado "El futuro de la manufactura" (Foresight, 2013). El gobierno chino emitió la estrategia "Hecho en China 2025" junto con el plan "Internet Plus" en 2015 y esta estrategia da prioridad a diez campos del sector manufacturero para acelerar la informatización y la industrialización en China (Li \& Lleytons, 2015).

La Comisión Europea lanzó la nueva Asociación Público-Privada (APP) en las fábricas del futuro (FOB) en 2014. Está comisión está dentro del marco del programa Horizonte 2020 que planea proporcionar casi 80 mil millones de euros en fondos durante 7 años (European Commission, 2016). En 2014, el gobierno de Corea del Sur anunció la "Innovación en la fabricación 3.0", que se priorizó en cuatro estrategias de propulsión y asignaciones para un nuevo salto de la fabricación coreana (Kang et al., 2016).

\subsection{Planes industriales.}

Desde el punto de vista de los planes industriales, AT\&T, Cisco, General Electric, IBM e Intel fundaron el "Industrial Internet Consortium (ICC)" en 2014 para canalizar y coordinar las prioridades y las tecnologías habilitadoras de la industria de Internet (Evans \& Annunziata, 2012). Mientras que otros proyectos relacionados con grandes empresas como Siemens, Hitachi, Bosch, Panasonic, Honeywell, Mitsubishi Electric, ABB, Schneider Electric y Emerson Electric también han realizado grandes inversiones en proyectos relacionados con IoT y CPS. Estas organizaciones han hecho de la cuarta revolución industrial uno de los temas más discutidos en muchas conferencias, foros y exposiciones de fabricación en los últimos años. Mientras tanto, un número creciente de centros de investigación, universidades y empresas también han participado y contribuido a este nuevo desafío a través de experimentos de laboratorio o aplicaciones industriales. Al observar todas estas contribuciones desde 
el mundo académico y la industria, se pueden encontrar intereses de investigación que la mayoría de ellos se enfocaron en revisar o estudiar un dominio. Como, por ejemplo, las tecnologías en la nube (Zhan et al., 2015), las redes inalámbricas industriales (Li et al., 2015), las tecnologías de integración (Thomas et al., 2016), la ingeniería virtual (Shafiq et al., 2015), los sistemas de información (Haddara \& Elragal, 2015), la prestación de servicios (Rennung et al., 2016) y tecnologías basadas en agentes (Adeyeri et al., 2015).

Uno de los elementos de la industria 4.0 es el IoT (Internet de las cosas) que ha favorecido una mejor calidad de la fluidez en los datos e información con la intervención de sensores de movimiento, cintas transportadoras, bases de datos, información de control de procesos en tiempo real y que cada uno de los elementos del sistema de producción a cumplir con los procedimientos de la organización en la entrega final del producto (Tiwari et al., 2018). Otra intervención que ha tenido la industria 4.0 es en áreas de cadenas de suministro que presentan modelos uniformes de información donde cada una de las personas involucradas en el proceso puede recopilar y procesar toda la información involucrada en el proceso de producción para tener una mejor planificación de la producción y estar más centrado en otras áreas para una mejor toma de decisiones (Grieco et al., 2017).

También conjuntamente en áreas de interés, planificación, estrategias y objetivos entre servicios al consumidor y optimización de procesos (López, 2016). Desarrollando una planificación y programación que permita la mejor toma de decisiones y automatización, generando y seleccionando a través de diferentes escenarios con el uso de métodos estadísticos en diferentes condiciones para hacer que la toma de decisiones sea más sólida (Caricato \& Grieco, 2017). Vale la pena mencionar que la capacitación continua en trabajadores involucrados en un proceso de fabricación debe pasar por las siguientes áreas de capacitación: didáctica, integradora e ingeniería, para el desarrollo de factores de aprendizaje. Un proceso de transformación adecuado y la intervención de la Industria 4.0 se reforzarán hacia una mejora continua en las áreas de ingeniería (Baena et al., 2017).

Actualmente tenemos conocimiento sobre los elementos de la industria 4.0 y cómo funciona cada uno de ellos. Sin embargo, es muy importante contar con un instrumento que pueda determinar el grado de madurez o las condiciones en que se encuentra cualquier organización, no solo para conocer las necesidades sino también para tomar las mejores decisiones e implementar estrategias hacia una mejora continua orientada a la industria 4.0 (Pozo, 2016).

\section{Esquema conceptual, variables e hipótesis de investigación.}

En este apartado se detallan los conceptos de las variables de estudio como la hipótesis a comprobar para la valoración, por parte de los usuarios, del modelo de relación y colaboración (MRC), así como su relación con los procesos de digitalización (ProDig). Esto implica la definición de las variables incluidas en el instrumento, así como la relación causal (teórica) a comprobar.

\subsection{Modelo de relación y colaboración (MRC).}

Esta variable está relacionada con la motivación y relación laboral no solo interna sino también externamente como lo son los proveedores, clientes, entidades financieras, etc. La salud es uno de los derechos fundamentales para todo ser humano y es considerado un factor insustituible que influye en el bienestar social, el desarrollo y la economía (Organización Mundial de la Salud, 2013). El analizar esta variable se obtiene información útil para la mejora continua en las condiciones laborales y los estándares de calidad en salud (Jiménez, 2004). Las hipótesis propuestas implican la valoración del modelo de relación y colaboración y los procesos de digitalización. 
$\mathrm{H}_{0 \mathrm{a}}$ : La valoración del modelo de relación y colaboración no tiene un efecto positivo en los procesos de digitalización.

$\mathrm{H}_{1 \mathrm{a}}$ : La valoración del modelo de relación y colaboración tiene un efecto positivo en los procesos de digitalización.

\subsubsection{Habilidades y competencias profesionales (ComProf)}

El entorno social y laboral influido por la revolución del nuevo conocimiento y el desarrollo de las nuevas tecnologías de informática y la comunicación ha llevado a la necesidad de estrechar la brecha hacia el mundo del trabajo (Tunning, 2007).

La UNESCO define competencia como la relación de comportamientos socio-afectivos y habilidades cognoscitivas, psicológicas, sensoriales y motoras que permiten llevar a cabo un desempeño, una función, una actividad o tarea. Desde esta perspectiva, lo importante no es la posesión de determinados conocimientos, sino el uso que se haga de ellos (Verdejo, 2006). Este criterio obliga a las organizaciones a replantear lo que comúnmente han considerado como formación.

\subsubsection{Formación digital (Fdig)}

De acuerdo con CAE Innovative Learning Solutions (2019) un 78\% de las empresas prevé invertir en formación digital en los próximos años. Sin embargo, uno de los inconvenientes con los que las empresas se enfrentan a la hora de considerar implantar la formación digital en su empresa, es el coste. Pero lo que muchas empresas olvidan es que, gracias a la formación digital, la empresa recibe un mayor retorno de inversión, por lo que invertir en la formación digital de sus empleados genera grandes beneficios.

La tecnología de la formación e-learning permite una pedagogía más variada, tanto por los dispositivos como por los recursos de formación. Aprender a explotar todas las posibilidades disponibles supone, sin duda, una aculturación y, por consiguiente, un cambio considerable en la conducta de los departamentos de formación (CAE-Innovate Learning Solutions).

\subsubsection{Procesos de digitalización (ProDig)}

La digitalización trata de las relaciones entre las personas, y entre las empresas y sus clientes, que obliga a las empresas a reinventarse y a revisar la estrategia y el modelo de negocio desde la óptica del cliente. La transformación digital en una empresa tiene como propósito convertirla en un nuevo ente plenamente conectado con el ecosistema digital, que le permita actuar de manera más ágil y con el punto de mira centrado en el cliente de un modo inteligente. Este proceso exige, además, un gran esfuerzo interno en la gestión del cambio para abordar con éxito este reto (Ffh-Fundació factor humá, 2019).

En esta investigación se propone evaluar las variables independientes (causas) habilidades y competencias profesionales y formación digital, con respecto a la variable dependiente (consecuencias) procesos de digitalización, a través de las siguientes hipótesis:

$\mathrm{H}_{0 \mathrm{~b}}$ : La valoración de las habilidades y competencias profesionales y formación digital no tiene un efecto positivo en los procesos de digitalización.

$\mathrm{H}_{1 \mathrm{~b}}$ : La valoración de las habilidades y competencias profesionales y formación digital tiene un efecto positivo en los procesos de digitalización. 


\section{Metodología.}

Para analizar el modelo conceptual propuesto, se emplearon técnicas como la prueba de fiabilidad de alfa de Cronbach que permite estimar la fiabilidad de un instrumento de medida a través de un conjunto de datos (Domínguez, 2012). La medida de la fiabilidad asume que los ítems medidos en escala tipo Likert miden una misma estructura y que están altamente correlacionadas (Matas, 2018; Welch \& Comer, 1988). Cuanto más cerca se encuentre el valor alfa de 1, mayor es la consistencia interna de los ítems analizados. El software estadístico empleado para los cálculos fue XLSTAT versión 2014 y para la validación del instrumento se utilizó SPSS v.25 (Statistical Package for the Social Sciences) ya que este sistema puede evaluar las variables dependientes e independientes con relación al uso y aplicación de tablas cruzadas y comparación de éstas. También se realizó un análisis de escalamiento métrico (o no métrico) multidimensional (EMM) que es un procedimiento multivariado de interdependencia cuyo fin es la identificación de las distancias o disimilitud en una matriz de datos, así como su representación gráfica en un espacio de pocas dimensiones, reproduciendo lo más fielmente posible las proximidades al interior de la muestra. Cuando las distancias que se utilicen para el análisis sean euclidianas el método de escalamiento será métrico; cuando no sea así los métodos utilizados serán no métricos (Greenacre \& Primicerio, 2013).

Los datos fueron recolectados de una muestra inicial de 20 personas ubicadas en la ciudad de Tampico, Tamaulipas (México), que manifestaron estar familiarizados con los procesos internos de la organización a la que pertenecen. La muestra fue seleccionada aleatoriamente aplicando un muestreo por conglomerados, teniendo en cuenta la ubicación de las organizaciones. La consulta fue realizada mediante una entrevista semiestructurada que presenta un mayor grado de flexibilidad que las estructuradas (Tabla 1), ya que comienzan con preguntas planificadas, que se pueden adaptar a los entrevistados. Su ventaja es la posibilidad de adoptar sujetos con enormes posibilidades para motivar al interlocutor, aclarar térmicas, identificar ambigüedades y reducir formalidades (Díaz et al., 2013).

Tabla 1. Fases de la entrevista.

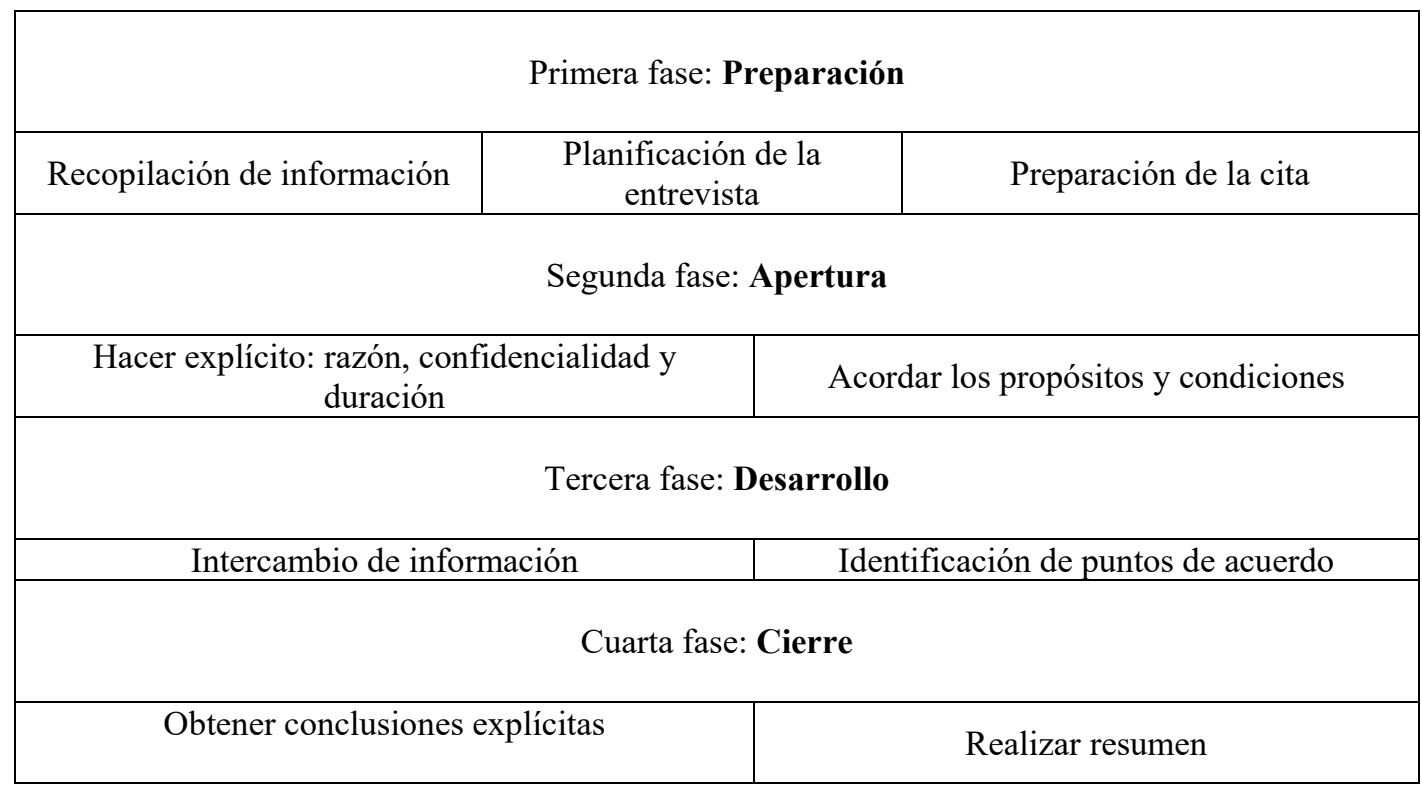

Fuente: Elaboración propia.

El instrumento fue diseñado a través de un análisis de los elementos que intervienen en la industria 4.0 hacia las organizaciones. Se estructuraron 16 aspectos a evaluar, distribuidas en forma secuencial en el instrumento, agrupadas por factores (Tabla 2).

El modelo completo se puede observar las variables (P1 a P16). Por cada pregunta, los encuestados podrían expresar su opinión a través de una escala tipo Likert de 7 puntos, donde 1 equivale 
a totalmente en desacuerdo y 7 totalmente de acuerdo. En orden a probar la consistencia interna del instrumento a través de los constructos o factores, se empleó el coeficiente de Alfa de Cronbach, arrojando como resultado un valor mínimo de 0,82 , superando el valor mínimo recomendado de 0,7 (Darren \& Mallery, 2003).

Tabla 2. Aspectos a evaluar y variables relacionadas con el instrumento de medición.

\begin{tabular}{|c|c|c|}
\hline Factor & Pregunta & Aspecto a evaluar \\
\hline \multirow{3}{*}{$\begin{array}{l}\text { Modelo de relación y } \\
\text { colaboración } \\
\text { (MRC) }\end{array}$} & P1 & $\begin{array}{l}\text { Grado de motivación de los empleados hacia la transformación a la } \\
\text { industria } 4.0\end{array}$ \\
\hline & $\mathrm{P} 2$ & $\begin{array}{lllll}\begin{array}{l}\text { Colaboración entre departamentos para la generación } \\
\text { oportunidades y eficiencia de las tecnologías }\end{array} & & & \\
\end{array}$ \\
\hline & $\mathrm{P} 3$ & $\begin{array}{l}\text { Relación de la organización con otros agentes (proveedores, clientes, } \\
\text { entidades financieras universidades, etc.) }\end{array}$ \\
\hline \multirow{3}{*}{$\begin{array}{l}\text { Habilidades y } \\
\text { competencias } \\
\text { profesionales } \\
\text { (ComProf) }\end{array}$} & $\mathrm{P} 4$ & Habilidades y competencias de los empleados \\
\hline & P5 & Habilidades y competencias digitales en relación a la industria 4.0 \\
\hline & P6 & $\begin{array}{l}\text { Dificultades existentes en los empleados respecto al uso de las } \\
\text { tecnologías en su desempeño laboral }\end{array}$ \\
\hline \multirow{3}{*}{$\begin{array}{l}\text { Formación digital } \\
\text { (Fdig) }\end{array}$} & P7 & $\begin{array}{l}\text { Inclusión de la formación digital sobre la industria } 4.0 \text { en su } \\
\text { organización }\end{array}$ \\
\hline & P8 & $\begin{array}{l}\text { Grado de formación actual de los empleados de acuerdo a las } \\
\text { necesidades de la industria } 4.0\end{array}$ \\
\hline & P9 & $\begin{array}{l}\text { Inversión en formación digital en su organización relacionado con } \\
\text { la industria } 4.0\end{array}$ \\
\hline \multirow{7}{*}{$\begin{array}{l}\text { Procesos de } \\
\text { digitalización } \\
\text { (ProDig) }\end{array}$} & $\mathrm{P} 10$ & Nivel de digitalización de los procesos de la organización \\
\hline & P11 & $\begin{array}{l}\text { Nivel de uso de habilitadores (conexión internet, apps, robótica, } \\
\text { impresión 3D, icloud, base de datos, otros) }\end{array}$ \\
\hline & P12 & $\begin{array}{l}\text { Herramientas digitales que generen flexibilidad en los procesos } \\
\text { productivos }\end{array}$ \\
\hline & P13 & Capacidad de la infraestructura tecnológica \\
\hline & P14 & Información en tiempo real de los sistemas de información \\
\hline & P15 & Grado de trazabilidad de la información \\
\hline & P16 & Calidad de la información generada por los sistemas de información \\
\hline
\end{tabular}
Fuente: Elaboración propia.

\section{Resultados.}

\subsection{Descripción de los datos.}

El factor de procesos de digitalización en las organizaciones (P13 y P16) son los resultados promedios más altos que hacen referencia a la infraestructura tecnológica y calidad de la información generada por los sistemas de información con un promedio de 5,0 puntos. La puntuación más baja se obtuvo en el aspecto de las dificultades existentes en los empleados respecto al uso de las tecnologías en su desempeño laboral (P6) con una media de 3,8. Los resultados descriptivos de las variables observables se registran en la Tabla 3. 
Tabla 3. Descripción de datos.

\begin{tabular}{|c|c|c|}
\hline Variable Observable & Media & Desviación Estándar \\
\hline P1 & 4,6 & 0,57 \\
\hline P2 & 4,5 & 0,60 \\
\hline P3 & 4,3 & 0,77 \\
\hline P4 & 4,7 & 0,86 \\
\hline P5 & 4,5 & 0,99 \\
\hline P6 & 3,8 & 1,19 \\
\hline P7 & 4,1 & 0,83 \\
\hline P8 & 4,5 & 0,67 \\
\hline P9 & 4,1 & 0,71 \\
\hline P10 & 4,6 & 0,65 \\
\hline P11 & 4,2 & 0,75 \\
\hline P12 & 4,3 & 1,37 \\
\hline P13 & 5,0 & 0,00 \\
\hline P14 & 4,1 & 1,3 \\
\hline P15 & 4,0 & 1,20 \\
\hline P16 & 5,0 & 0,51 \\
\hline
\end{tabular}

Fuente: Elaboración propia.

\subsection{Fiabilidad y validez del instrumento.}

El modelo o esquema propuesto de tipo reflectivo presenta niveles satisfactorios de validez y fiabilidad (Prieto \& Delgado, 2010; Vizcaíno et al., 2014). Los constructos mantienen características de homogeneidad y unidimensionalidad mediante la prueba de análisis de factor con rotación de tipo varimax que se encarga de considerar el aumento de la varianza de las cargas factoriales al cuadrado de cada factor y que los resultados de las cargas tiendan a acercarse a 1 y mientras que otras se aproximan a 0 , se obtienen una pertenencia más clara e inteligible de cada variable al factor. Para la validez convergente-, se calculó la varianza promedio extraída (o AVE, por Average Variance Extracted) por constructo, obteniendo valores superiores a 0,66 , estando por encima de 0,5 , que es el valor mínimo recomendado mostrado en la tabla 4 (Hair et al., 2016; Monteiro et al., 2016).

Tabla 4. Matriz de rotación Varimax y AVE por constructo.

\begin{tabular}{|c|c|c|}
\hline Factor & Matriz de componente rotado (Varimax) & AVE \\
\hline $\begin{array}{c}\text { Modelo de relación y } \\
\text { colaboración (MRC) }\end{array}$ & 0,88 & 0,66 \\
\hline $\begin{array}{c}\text { Habilidades y competencias } \\
\text { profesionales (ComProf) }\end{array}$ & 0,68 & 0,74 \\
\hline Formación Digital (Fdig) & 0,77 & 0,69 \\
\hline $\begin{array}{c}\text { Procesos de digitalización } \\
\text { (proDig) }\end{array}$ & 0,66 & 0,72 \\
\hline
\end{tabular}

Fuente: Elaboración propia. 
Por otra parte, la estimación del factor de inflación de la varianza (o VIF, por Variance Inflation Factor) indica que no existe multicolinealidad entre pares de constructos o factores independientes (véase Tabla 5), obteniendo un valor máximo de 1,98 (Chen, 2012; Montgomery, 2011).

Tabla 5. Tolerancia y VIF por constructo.

\begin{tabular}{|c|c|c|}
\hline Factor & Tolerancia & VIF \\
\hline Modelo de relación y colaboración (MRC) & 0,53 & 1,87 \\
\hline Habilidades y competencias profesionales (ComProf) & 0,50 & 1,98 \\
\hline Formación digital (Fdig) & 0,72 & 1,38 \\
\hline
\end{tabular}

Fuente: Elaboración propia.

Por último, la validez discriminante aplicada al modelo de cada constructo es independiente de los otros constructos, excepto de aquél que es teóricamente asociado en el esquema propuesto (Henseler et al., 2015), comparando cada coeficiente de regresión y carga o efecto individual con los efectos cruzados de cada variable observada para los demás constructos (Tabla 6).

Tabla 6. Matriz de efectos cruzados.

\begin{tabular}{|c|c|c|c|c|}
\hline & MRC & ComProf & FDig & ProDig \\
\hline P1 & $\mathbf{0 , 9 2 7}$ & $-0,682$ & $-0,254$ & 0,762 \\
\hline P2 & $\mathbf{0 , 1 8 1}$ & 0,040 & $-0,126$ & 0,148 \\
\hline P3 & $\mathbf{0 , 3 3 7}$ & $-0,540$ & $-0,044$ & 0,277 \\
\hline P4 & 0,387 & $\mathbf{- 0 , 2 6 2}$ & $-0,057$ & 0,208 \\
\hline P5 & 0,711 & $\mathbf{- 0 , 7 8 5}$ & $-0,071$ & 0,626 \\
\hline P6 & $-0,461$ & $\mathbf{0 , 9 2 3}$ & 0,205 & $-0,735$ \\
\hline P7 & $-0,050$ & 0,252 & $\mathbf{0 , 4 3 2}$ & $-0,261$ \\
\hline P8 & 0,238 & 0,104 & $\mathbf{- 0 , 4 4 1}$ & 0,266 \\
\hline P9 & $-0,062$ & $-0,098$ & $\mathbf{- 0 , 2 2 8}$ & 0,138 \\
\hline P10 & 0,051 & $-0,341$ & 0,363 & $\mathbf{0 , 0 1 3}$ \\
\hline P11 & $-0,046$ & $-0,177$ & 0,139 & $\mathbf{- 0 , 0 0 4}$ \\
\hline P12 & 0,341 & $-0,261$ & $-0,579$ & $\mathbf{0 , 5 3 2}$ \\
\hline P13 & 0,094 & $-0,192$ & 0,471 & $\mathbf{- 0 , 0 8 3}$ \\
\hline P14 & 0,582 & $-0,350$ & $-0,490$ & $\mathbf{0 , 6 4 0}$ \\
\hline P15 & $-0,330$ & 0,622 & 0,304 & $\mathbf{- 0 , 5 6 6}$ \\
\hline P16 & 0,428 & $-0,727$ & 0,288 & $\mathbf{0 , 3 9 1}$ \\
\hline
\end{tabular}

Fuente: Elaboración propia.

\subsection{Análisis del modelo estructural.}

En la tabla 7 se pueden observar los pesos externos y las correlaciones se agrupan en dos tablas grandes. $\mathrm{Si}$ estudiamos las correlaciones entre las variables manifiestas y las variables latentes, podemos observar que las variables manifiestas MRC1 tienen un mayor efecto sobre el modelo de relación y colaboración que MRC2 y MRC3. Esta tabla permite ver el impacto de cada variable manifiesta asociada en su variable latente asociada. En la figura 1, se muestra el diagrama con los coeficientes por ruta y las varianzas explicadas. 
Tabla 7. Modelo externo de variables latentes.

\begin{tabular}{|c|c|c|c|c|c|}
\hline $\begin{array}{c}\text { Variable } \\
\text { latente }\end{array}$ & $\begin{array}{c}\text { Variables } \\
\text { manifiestas }\end{array}$ & $\begin{array}{c}\text { Peso } \\
\text { externo }\end{array}$ & $\begin{array}{c}\text { Correlación de } \\
\text { cargas } \\
\text { estandarizadas }\end{array}$ & $\begin{array}{c}\text { Límite } \\
\text { inferior }\end{array}$ & $\begin{array}{c}\text { Límite } \\
\text { superior }\end{array}$ \\
\hline \multirow{3}{*}{ MRC } & MRC1 & 1,038 & 0,927 & $-1,526$ & 1,563 \\
\cline { 2 - 6 } & MRC2 & 0,377 & 0,181 & $-1,078$ & 1,255 \\
\cline { 2 - 6 } & MRC3 & $-0,088$ & 0,337 & $-1,302$ & 2,298 \\
\hline \multirow{3}{*}{ ComProf } & ComProf1 & $-0,069$ & $-0,262$ & $-1,320$ & 1,109 \\
\cline { 2 - 6 } & ComProf2 & $-0,401$ & $-0,785$ & $-1,657$ & 1,486 \\
\cline { 2 - 6 } & ComProf3 & 0,722 & 0,923 & $-0,816$ & 1,661 \\
\hline \multirow{4}{*}{ FDig } & FDig1 & 1,111 & 0,432 & $-1,328$ & 2,144 \\
\cline { 2 - 6 } & FDig2 & $-1,101$ & $-0,441$ & $-2,736$ & 2,745 \\
\cline { 2 - 6 } & FDig3 & $-0,152$ & $-0,228$ & $-1,756$ & 0,951 \\
\hline \multirow{4}{*}{ ProDig } & ProDig1 & $-0,390$ & 0,013 & $-34,659$ & 11,198 \\
\cline { 2 - 6 } & ProDig2 & $-0,113$ & $-0,004$ & $-17,901$ & 28,416 \\
\cline { 2 - 6 } & ProDig3 & 0,304 & 0,532 & $-42,657$ & 50,752 \\
\cline { 2 - 6 } & ProDig4 & $-0,045$ & $-0,083$ & $-4,674$ & 15,793 \\
\cline { 2 - 6 } & ProDig5 & 0,619 & 0,640 & $-69,899$ & 51,496 \\
\cline { 2 - 6 } & ProDig6 & $-0,377$ & $-0,566$ & $-15,083$ & 8,516 \\
\cline { 2 - 5 } & ProDig7 & 0,588 & 0,391 & $-6,261$ & 12,274 \\
\hline
\end{tabular}

Fuente: Elaboración propia.

Figura 1. Diagrama de coeficientes y varianzas explicadas.

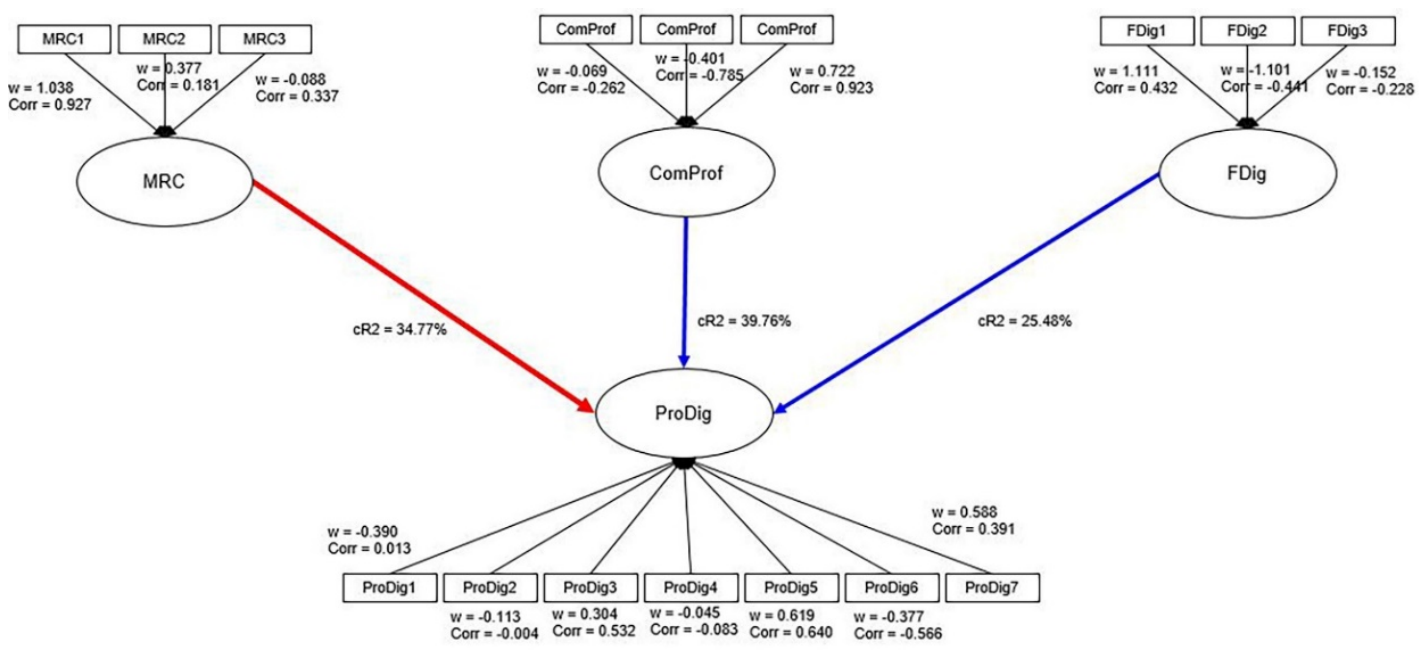

Fuente: Elaboración propia.

Por último, en este trabajo se realizó un resumen de resultados (Tabla 8) que muestra los valores percibidos y el porcentaje de contribución al $\mathrm{R}^{2}$ de la variable de procesos de digitalización.

Tabla 8. Impacto y contribución de las variables a procesos de digitalización.

\begin{tabular}{|c|c|c|c|}
\hline Indicador & MRC & FDig & ComProf \\
\hline Correlación & 0,822 & $-0,603$ & $-0,797$ \\
\hline Path coefficient & 0,397 & $-0,397$ & $-0,469$ \\
\hline Correlación * Coeficiente & 0,326 & 0,239 & 0,373 \\
\hline Contribución al R ${ }^{2}(\%)$ & 34,765 & 25,476 & 39,759 \\
\hline \% acumulado & 34,765 & 60,241 & 100,000 \\
\hline
\end{tabular}

Fuente: Elaboración propia. 
Figura 2. Diagrama de coeficientes y varianzas explicadas.

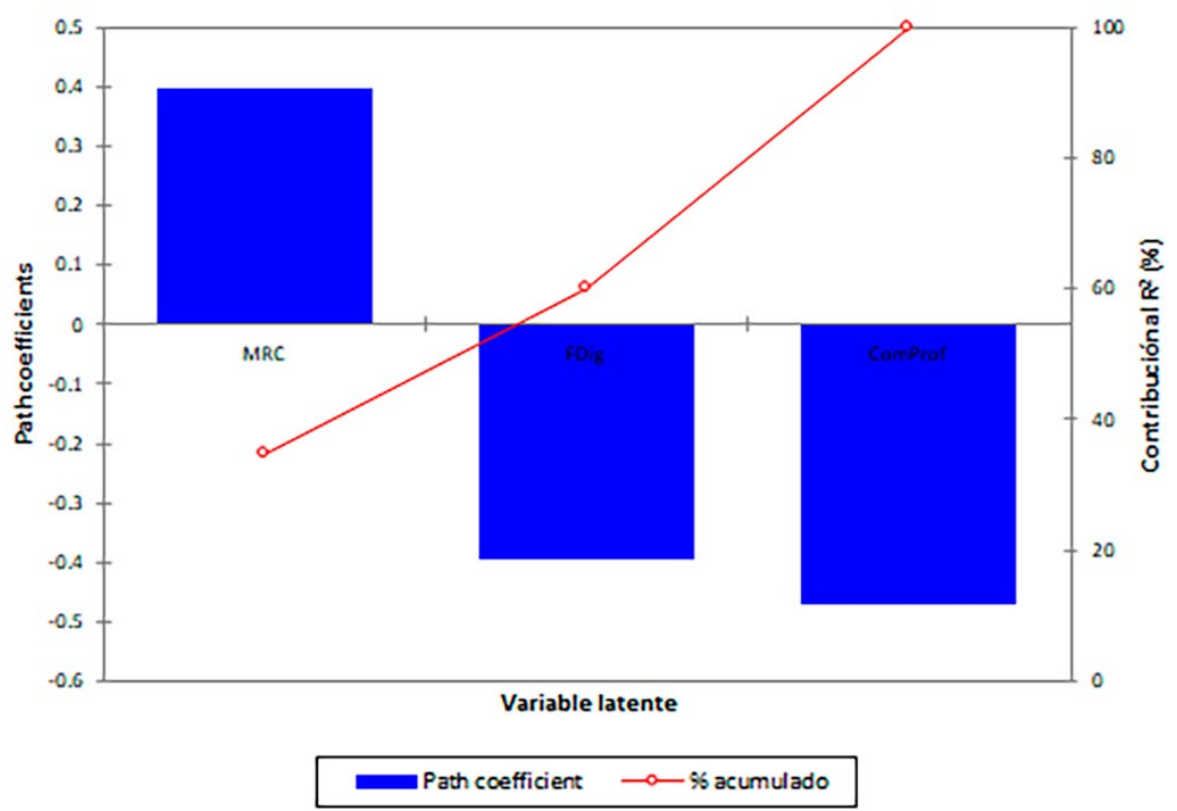

Fuente: Elaboración propia.

En la Figura 2 la variable MRC se relaciona de forma positiva con la variable de procesos de digitalización. La variable fDig y ComProf tienen una relación negativa con la variable de procesos de digitalización y es debido a que la innovación y adopción de nuevos procesos digitales requieren que los niveles de formación y competencias profesionales aumenten de una manera rápida y que no se está realizando. El grado de motivación aumenta con el incremento en la digitalización de los procesos.

De acuerdo con los resultados, el modelo de relación y colaboración tuvo un efecto positivo en los procesos de digitalización, por tanto, se aceptó esta hipótesis, mientras que la valoración de las habilidades y competencias profesionales y formación digital no tuvo efecto positivo en los procesos de digitalización, rechazándose este planteamiento.

\section{Discusión y conclusiones.}

El objetivo principal del artículo se concentró en la valoración, por parte de los usuarios, de la satisfacción laboral respecto a los procesos de digitalización en las organizaciones a la que pertenecen en Tampico, Tamaulipas (México) a través de la aplicación de la técnica de regresión de mínimos cuadrados parciales (PLS), que permitiera identificar los factores determinantes de la satisfacción laboral y la digitalización. Para este estudio, se consideraron las variables modelo de relación y colaboración, habilidades y competencias profesionales, formación digital y procesos de digitalización.

Los resultados del modelo propuesto muestran que existe una relación de medias entre el aspecto de infraestructura tecnológica y el de calidad de la información generada por los sistemas de información. También se realizó una matriz de rotación de tipo varimax para determinar el nivel de pertenencia de los factores y el cálculo de la varianza promedio extraída (AVE) donde el resultado de consistencia de constructo fue aceptado.

Por otra parte, la estimación del factor de inflación de la varianza (o VIF, por Variance Inflation Factor) indica que no existe multicolinealidad entre pares de constructos o factores independientes.

Por último, es necesario tener en cuenta que, para este tipo de estudios, deben considerarse las limitaciones de aceptabilidad por parte de las organizaciones ya que algunas presentan desconocimiento 
sobre los elementos que integran la industria 4.0 y es necesario aplicar el instrumento a personas que cuenten con años de experiencia laboral y que estén completamente relacionados con los procesos industriales y digitales de su organización. Se propone para investigaciones futuras la inclusión de nuevos elementos relacionados con la industria 4.0 en el ámbito de los negocios y cadena de valor para ir conociendo la perspectiva de las áreas gerenciales y su relación con otras organizaciones.

Las repercusiones de esta investigación relacionado con la industria 4.0 es que las organizaciones optimicen sus sistemas de fabricación, acorten el ciclo de desarrollo de nuevos productos, reduzcan los costos de fabricación y cuenten con procesos productivos totalmente integrados y automatizados, con máquinas capaces de autoadministrarse y mantenerse. Igualmente, la Industria 4.0 podría generar cambios en la fuerza laboral, requiriendo nuevas capacidades, aptitudes y roles, porque este concepto supone un cambio de mentalidad muy importante y una gran transformación en las empresas. Es importante no quedarse al margen porque la Cuarta Revolución Industrial supondrá una fuente de competitividad para las industrias occidentales tanto en costes de mano de obra, energía, así como en niveles de compromiso social.

\section{Referencias}

Adeyeri, M., Mpofu, K., \& Tijani, O. (2015). Integration of Agent Technology into Manufacturing Enterprise. A Review and Platform for Industry 4.0. In 2015 International Conference on Industrial Engineering and Operations Management (IEOM), 110. Dubai: IEEE.

Atzori, L., Antonio, L., \& Morabito, G. (2010). The Internet of Things: A survey. Journal Computer Networks, 54(15), 2287-2805. DOI: 10.1016/j.comnet.2010.05.010.

Baena, F., Guarin, A., Mora, J., Sauza, J., \& Retat, S. (2017). Learning Factory: The Path to Industry 4.0. Journal Procedia Manufacturing, 9, 73-80. DOI: 10.1016/j.promfg.2017.04.022.

Belvedere, V., Grando, A., \& Bielli, P. (2013). A Quantitative Investigation of the Role of Information and Communication Technologies in the Implementation of a Product-service System. International Journal of Production Research, 51(2), 1-17. DOI: 10.1080/00207543.2011.648278.

Cae-Innovative Learning Solutions. (2019). ¿Por qué invertir en la formación digital de sus empleados? Nota descriptiva. Recuperado de https://www.cae.net/es/ventajas-de-la-formaciondigital.

Caricato, P., \& Grieco, A. (2017). An application of Industry 4.0 to the production of packaging films. Journal Procedia Manufacturing, 11, 949-956. DOI: 10.1016/j.promfg.2017.07.199.

Chen, J. (2012). Advances in Hospitality and Leisure (volume 15). USA: Advances in Hospitality and Leisure.

Consell national de l'industrie. (2013). The New Face of Industry in France. Paris: French National Industry Council.

Darren, G., \& Mallery, P. (2003). SPSS for Windows step by step: A simple guide and reference. 11.0 update (4 ed.). Boston, MA: Allyn \& Bacon.

Díaz, L., Torruco, U., Mildred, M., \& Varela M. (2013). La entrevista, recurso flexible y dinámico. Revista de Investigación en Educación Médica, 2(7), 162-167. DOI: 10.1016/S20075057(13)72706-6 
Domínguez, S. (2012). Propuesta para el cálculo de alfa ordinal y theta de armor. Revista de investigación en Psicología, 15(1), 213-217. DOI: 10.15381/rinvp.v15i1.3684.

Drath, R., \& Horch, A. (2014). Industrie 4.0: Hit or Hype? IEEE Industrial Electronics Magazine, 8(2), 56-58. DOI: 10.1109/MIE.2014.2312079.

European Commission. (2016). Factories of the Future PPP: Towards Competitive EU Manufacturing. Bruxelles: European Commission.

Evans, P., \& Annunziata, M. (2012). Industrial Internet: Pushing the Boundaries of Minds and Machines. Boston, MA: General Electric.

Ffh-Fundació factor humá (2019). La digitalización acelera el cambio cultural. Nota descriptiva. Recuperado de https:/factorhuma.org/es/actualitat/noticias/12711-articulo-de-opinion-ladigitalizacion-acelera-el-cambio-cultural

Foresight (2013). The Future of Manufacturing: A New Era of Opportunity and Challenge for the UK, London: UK Government Office for Science.

Greenacre, M., \& Primicerio, R. (2013). Multivariate Analysis of Ecological Data. Bilbao: Fundación BBVA.

Grieco, A., Caricato, P., Gianfreda, D., Pesce, M., Rigon, V., Tregnaghi, L., \& Voglino, A. (2017). An Industry 4.0 case study in fashion manufacturing. Journal Procedia Manufacturing, 11, 871-877. DOI: $10.1016 /$ j.promfg.2017.07.190.

Haddara, M., \& Elragal, A. (2015). The Readiness of ERP Systems for the Factory of the Future. Journal Procedia Computer Science, 74, 721-728. DOI: 10.1016/j.procs.2015.08.598.

Hair, J., Hult, G., Ringle, C., \& Sarstedt, M. (2016). A Primer on Partial Least Squares Structural Equation Modeling (PLS-SEM), (2ed). Nueva York: SAGE Publications.

Henseler, J., Ringle, C., \& Sarstedt, M. (2015). A new criterion for assessing discriminant validity in variance-based structural equation modeling. Journal of the Academy of Marketing Science, 43(1), 115-135. DOI: $10.1007 / \mathrm{s} 11747-014-0403-8$.

Jiménez, R. (2004). Indicadores de calidad y eficiencia de los servicios hospitalarios. Una mirada actual. Revista Cubana de Salud Pública, 30(1), 17-36.

Kagermann, H., Wolfgang, W., \& Helbig, J. (2013). Recommendations for Implementing the Strategic Initiative INDUSTRIE 4.0. Berlin: Industrie 4.0, Working Group of Acatech.

Kang, H.S., Lee, J.Y., \& Kim, H. (2016). Sang Smart Manufacturing: Past Research, Present Findings, and Future Directions, International Journal of Precision Engineering and Manufacturing-Green Technology, 3, 111-128. DOI: https: 10.1007/s40684-016-0015-5.

Khaitan, S., \& Mccalley, J. (2015). Design Techniques and Applications of Cyberphysical Systems: A Survey. IEEE Systems Journal, 9(2), 1-16. DOI: 10.1109/JSYST.2014.2322503.

Li, K., \& Lleytons. (2015). Made in China 2025. Beijing: State Council of China. 
Li, X., Di, L., Jiafu, W., Vasilakos, A., Lai, C., \& Wang, S. (2015). A Review of Industrial Wireless Networks in the Context of Industry 4.0, Journal Wireless Networks, 23, 23-41. DOI: 10.1007/s11276-015-1133-7.

López, D. (2016). Análisis de Casos de Estudio sobre Industria 4.0 y Clasificación según Sectores de Actividad y Departamentos Empresariales. España: Universidad Politécnica de Valencia.

Matas, A. (2018). Diseño del formato de escalas tipo Likert: un estado de la cuestión. Revista Electrónica de Investigación Educativa, 20(1), 38-49. DOI: https: 10.24320/redie.2018.20.1.1347

Maynard, A. (2015). Navigating the Fourth Industrial Revolution. Journal Nature Nanotechnology, 10(12), 1005-1006. DOI: 10.1038/nnano.2015.286.

Monteiro, D., Marinho, D., Moutao, J., Couto, N., Antunes, R., \& Cid, L. (2016). Adaptation and validation of the Portuguese version of Basic Psychological Needs Exercise Scale (BPNESp) to the sport domain and invariance across football and swimming. Journal Montricidade, 12(4), 51-61. DOI: $10.6063 /$ motricidade.9372.

Montgomery, V. (2011). Introducción al análisis de regresión lineal. (3ed). México. Editorial Patria.

National Research Foundation. (2016). Research, Innovation and Enterprise (RIE) 2015. Singapore: Prime Minister's Office of Singapore.

Organización Mundial de la Salud (2013). Derecho a la salud. Nota descriptiva n.23 [consultado 01 de abril 2019]. Recuperado de http://www.who.int/mediacentre/factsheets/fs323/es/

Pozo, M. (2016). 4 herramientas para dar paso a la industria 4.0. Recuperado de https://ciudadesdelfuturo.es/4-herramientas-digitales-industria-4-0.php.

Prieto, G., \& Delgado, A. (2010). Fiabilidad y Validez. Revista Papeles del Psicólogo, 31(1), 67-74. DOI: $10.4067 / \mathrm{S} 0718-09342002005100014$.

Reif, R., Liveris, A., \& Ann, S. (2014). Report To The President Accelerating U.S. Advanced Manufacturing. Washington, DC: The President's Council of Advisors on Science and Technology.

Rennung, F., Luminosu, C., \& Draghici, A. (2016). Service Provision in the Framework of Industry 4.0. Journal Procedia - Social and Behavioral Sciences, 221, 372-377. DOI: $10.1016 /$ j.sbspro.2016.05.127.

Ridgway, K., Clegg, C., Williams, D., \& Hourd, P. (2013). The Factory of the Future. London: Government Office for Science.

Shafiq, S.Y., Sanim, C., Szczerbicki, E., \& Toro, C. (2015). Virtual Engineering Object/Virtual Engineering Process: A specialized form of Cyber Physical System for Industrie 4.0. Journal Procedia Computer Science, 60(1), 1146-1155. DOI: 10.1016/j.procs.2015.08.166.

Thomas, B., Riedl, M., Thron, M., \& Diedrich, C. (2016). Integration of Classical Components into Industrial Cyber-Physical Systems. Journal Proceedings of the IEEE, 104(5), 947-959. DOI: 10.1109/JPROC.2015.2510981. 
Tiwari, S., Wee, H., \& Daryanto, Y. (2018). Big data analytics in supply chain management between 2010 and 2016: Insights to industries. Journal Computers \& Industrial Engineering, 115, 319-330. DOI: $10.1016 /$ j.cie.2017.11.017.

Tunning. (2007). Reflexiones y perspectivas de la educación superior en América Latina. Informe final. Proyecto Tunning-America Latina. España, Bilbao: Universidad de Deusto, Universidad de Groningen.

Verdejo, P. (2006). Modelo para la educación y evaluación por competencias (MECO), Aseguramiento de la Calidad en la Educación y en el Trabajo,155-195. Editorial: Universidad Autónoma de Baja California, México.

Vizcaíno, A., Manzano, M., \& Casas, G. (2015). Validez de constructo y confiabilidad del cuestionario de creencias epistemológicas sobre las matemáticas en alumnos de secundaria básica. Revista Colombiana de Psicología, 24(2), 301-316. DOI: 10.15446/rcp.v24n2.43974.

Welch, S., \& Comer, J. (2018). Quantitative Methods for Public Administration: Techniques and Applications. USA: Editorial Waveland Pr Inc.

Zhan, Z., Xiao, L., Yue, G., Jun, Z., Henry, S., \& Yun, L. (2015). Cloud Computing Resource Scheduling and a Survey of Its Evolutionary Approaches, Journal ACM Computing Surveys, 47(4), 1-33. DOI: $10.1145 / 2788397$. 